Álvarez-Muñoz, J.S. \& Hernández-Prados, M.A. (2022). El desarrollo de las capacidades desde el ocio familiar. Revista Electrónica Interuniversitaria de Formación del Profesorado, 25(1), 35-48.

DOI: https://doi.org/10.6018/reifop.502551

\title{
El desarrollo de las capacidades desde el ocio familiar
}

José Santiago Álvarez Muñoz y Ma Ángeles Hernández Prados

Universidad de Murcia

\section{Resumen}

Actualmente existe una tendencia educativa centrada en el desarrollo de las capacidades del ser humano, tanto cognitivas como no cognitivas, que trata de identificar los aspectos que contribuyen a su adquisición y consolidación. En esta línea, se pretende conocer el potencial de ocio familiar que le atribuyen los adolescentes para el desarrollo de las capacidades de aprendizaje. Para ello se aplicó el cuestionario final a 1054 estudiantes de 9 centros murcianos de educación secundaria, y se analiza la variable capacidades del ocio familiar a partir de los resultados del análisis factorial exploratorio que permite diferenciar en tres tipos de capacidades: académicas, organizativas y relacionales, observando el predominio de las dos últimas sobre la primera. Los resultados muestran que los adolescentes de centros concertados-privados atribuyen más capacidades al ocio familiar que los que cursan en los públicos. Se concluye la necesidad de promover un ocio familiar con fines educativos en la mejora de la creatividad, atención, memoria e inteligencia emocional, aproximando lo académico a un tiempo de disfrute familiar, libre de tensiones y de estrés.

\section{Palabras clave}

Ocio; familia; capacidades; adolescentes.

\section{Capacity building through family leisure activities}

\section{Abstract}

There is currently an educational trend focused on the development of human abilities, both cognitive and non-cognitive, which seeks to identify the aspects that contribute to their acquisition and consolidation. Along these lines, the aim is to find out the potential of family leisure time ascribed by adolescents for the development of learning skills. To this end, the

\section{Contacto:}

Mª́ngeles Hernández Prados, mangeles@um.es 
final questionnaire was applied to 1054 students from 9 secondary schools in Murcia, and the variable family leisure skills is analysed on the basis of the results of the exploratory factor analysis which allows differentiation into three types of skills: academic, organisational and relational, observing the predominance of the last two over the first. The results show that adolescents in private-subsidised schools attribute more skills to family leisure than those in public schools. We conclude that there is a need to promote family leisure for educational purposes in order to improve creativity, attention, memory and emotional intelligence, bringing academic activities closer to a time of family enjoyment, free of tension and stress.

\section{Key words}

Leisure; family; skills; teenagers.

\section{Introducción}

Toda educación persigue el desarrollo-potencialización-capacitación de la persona, en un proceso continuo e inacabable de construcción que comienza desde el momento en el que nacemos y depende del contexto y circunstancias que nos envuelven. En este proceso la capacidad de aprendizaje para acumular conocimiento tácito y explícito, así como reflexionar regularmente sobre las rutinas de aprendizaje, que se lleva a cabo absorbiendo, asociando, recombinando, creando y experimentando con el conocimiento (Boukamel et al., 2019) adquiere un papel relevante.

Resulta difícil hablar de capacidad en singular y sin adjetivar (de aprendizaje, innovación, tecnológica, organizacional, etc), además de aglutinar diversas capacidades en cada una de las categorías ejemplificadas. Así como la capacidad de innovación integra la capacidad de aprendizaje, conectiva, ambidestreza, seguimiento de riesgos, liderazgo y tecnológica (Boukamel et al., 2019), la capacidad de aprendizaje, a su vez, hace mención a la creatividad, el procesamiento de información, la atención o la reflexión (Gallego y Araque, 2019), y guarda relación con la capacidad emprendedora, organizativa e innovadora (Fernández-Mesa et al, 2012).

En cuanto a los aspectos que favorecen el desarrollo de las capacidades de aprendizaje en la adolescencia, las variables escolares (titularidad del centro, etapa escolar, metodologías docentes, rendimiento académico, etc.) y familiares (estudios y estilos parentales, ocupación laboral, nivel socioeconómico, etc.) han sido las contempladas tradicionalmente (Alves et al. 2017; Martín et al., 2018; Melton y Ellis, 2019). Sin embargo, están emergiendo otros aspectos que contribuyen al desarrollo de capacidades como el campo de actuación del ocio, y más específicamente al familiar, que cuenta con un amplio recorrido a pesar de ser una línea de investigación reciente (Hodge et al., 2017; Trussell et al., 2017).

Las nuevas tendencias en el estudio del ocio familiar no se limitan a determinar la frecuencia o la duración de las actividades realizadas, más bien pretenden analizar su potencial como fuente de desarrollo, para lo que se requiere entrar de lleno en el contenido y los recursos utilizados (McCormick et al., 2020), respetando la autonomía de cada familia en la elección de las actividades de ocio de acuerdo con sus creencias, objetivos, intereses, comprensión del mundo del menor, capacidades y conocimientos a transmitir, etc. (Costa et al., 2020). Aunque los beneficios que se le atribuyen dependerán en gran medida del tipo de actividad a realizar, aquellos que tiene que ver con lo relacional-convivencial y emocional-satisfactorio son ampliamente generalizables. 
Estudios como el de Costa et al. (2020) permitieron comprobar los efectos positivos que diversas actividades recreativas desarrolladas en el marco de la experiencia colectiva familiar promovían en la mejora de la convivencia, el diálogo y bienestar, además de hacer posibles enfoques que enriquecen las relaciones interpersonales. Compartir experiencias de ocio con la familia, además de crear patrones de ocio deseables, tiene consecuencias positivas en la organización de sus miembros y en las relaciones de apego o afecto, incrementando la confianza, cohesión y capacidad de reacción al cambio (Varela y Maroñas, 2019).

De igual modo, cabe mencionar la importancia del ocio familiar como contexto relacional a través del cual se adquieren pautas, habilidades y normas para poder extrapolar a otros espacios externos al de la familia (Melton, 2017). Este potencial relacional del ocio familiar ha sido constatado, especialmente por las madres, incluso en contextos en los que habitan menores con diversidad funcional (Trussell et al. ,2017). Por otro lado, la alternativa de ocio familiar relacionada con los museos ha estado siempre relacionada con el desarrollo de capacidades cognitivas-académicas, pero recientemente se le ha atribuido beneficios referidos al fortalecimiento de los lazos familiares, el apego a la comunidad, la conciencia cultural, la restauración y el crecimiento personal (Zhou et al., 2019).

Pero sin lugar a dudas, la relación más analizada ha sido la existente entre ocio familiar y rendimiento académico (Hernández-Prados y Álvarez-Muñoz, 2019; Park y Lee, 2020). Esta línea perdura, aunque no se limita al estudio de las capacidades puramente académicas, como la memoria o la atención, sino que aparece un nuevo modelo de ocio familiar novedoso y diversificado que fomenta e incentiva el pensamiento creativo de los hijos y los padres (Martín Quintana et al., 2018) y representa un rol mediador en el desarrollo de la inteligencia emocional (Hodge et al., 2017). La adquisición de estas habilidades depende en gran medida del tiempo de dedicación y el grado de participación que tenga el menor en la planificación, preparación y desarrollo de la actividad ideada (Townsend et al., 2017).

Atendiendo a la fundamentación teórica expuesta en la introducción, desde el enfoque de la epistemología sistémica en el que todo esta relacionado, se evidencia la complejidad del desarrollo de capacidades en los seres humanos, mediante la construcción de escenarios educativos en los que el ocio familiar constituye un entorno más de aprendizaje y desarrollo que cuenta con gran potencial educativo, pero escasamente considerado en investigación. Aunque teóricamente se sustentan un posicionamiento complejo y holístico, no es incompatible y nada despreciable el estudio de partes específicas, planteando problemas empíricos que permitan simplificar (Boukamel et al., 2019).

En este sentido, la presente investigación se plantea como propósito principal conocer la percepción que tienen los adolescentes que se encuentran matriculados en centros de educación secundaria sobre el potencial educativo atribuible al ocio familiar con respecto al desarrollo de determinadas capacidades de aprendizaje. Concretamente, se proponen los siguientes objetivos específicos: 1) analizar, a nivel global, la atribución del desarrollo de capacidades al ocio familiar por parte de los adolescentes, así como 2) observar dicha participación en función de las variables sociodemográficas de índole personal, escolar y familiar, centrándonos especialmente en la titularidad del centro.

\section{Metodología}

Estudio de corte cuantitativo, no experimental y descriptivo-exploratorio que busca, en base a unos objetivos preestablecidos, examinar una realidad concreta que no ha sido alterada previamente. Se realiza una evaluación de un contexto específico a partir de la recolección 
de datos por medio de la cumplimentación de un cuestionario, obteniendo resultados que pueden ser generalizados y extrapolados a otros contextos (Hernández et al., 2010).

\section{Muestra}

Los participantes fueron seleccionados bajo un muestreo intencional atendiendo a dos criterios de inclusión: (a) estudiantes de Educación Secundaria (b) matriculado en un colegio/instituto ubicado en la comunidad autónoma de la Región de Murcia. Los 1054 adolescentes de Educación Secundaria Obligatoria de nueve centros educativos la Región de Murcia (siete públicos y dos concertados-privados) constituyen el tamaño muestral con un nivel de confianza del $95 \%(Z=1,96)$ y un margen de error del $5 \%$ en base a los datos de escolarización aportados por la Consejería de Educación de la Región de Murcia. Los participantes cumplimentaron voluntaria y anónimamente el cuestionario tras la autorización de los progenitores mediante un documento de consentimiento informado, atendiendo a la norma 8.2 de la APA y los parámetros éticos de la investigación educativa. En la Tabla 1 se recoge, a modo síntesis, los datos sociodemográficos de los estudiantes participantes.

\section{Tabla 1}

Distribución muestral de los participantes

\begin{tabular}{|c|c|c|c|}
\hline Variable & Categoría & Frecuencia & Porcentaje \\
\hline \multirow{2}{*}{ Sexo } & Hombre & 544 & $51,6 \%$ \\
\hline & Mujer & 510 & $48,4 \%$ \\
\hline \multirow{3}{*}{ Edad } & Menor de 13 años & 472 & $44,8 \%$ \\
\hline & De 14 a 16 años & 553 & $52,5 \%$ \\
\hline & Más de 16 años & 29 & $2,8 \%$ \\
\hline \multirow{4}{*}{ Curso } & $1^{\circ}$ E.S.O & 282 & $26,8 \%$ \\
\hline & $2^{\circ}$ E.S.O & 217 & $20,6 \%$ \\
\hline & $3^{\circ}$ E.S.O & 276 & $26,2 \%$ \\
\hline & $4^{\circ}$ E.S.O & 278 & $26,4 \%$ \\
\hline \multirow{2}{*}{ Titularidad } & Público & 814 & $77,2 \%$ \\
\hline & Concertado & 240 & $22,8 \%$ \\
\hline \multirow{2}{*}{ Nacionalidad } & Española & 1024 & $97,2 \%$ \\
\hline & Extranjera & 30 & $2,8 \%$ \\
\hline \multirow{4}{*}{ Tipo de familia } & Nuclear & 814 & $77,3 \%$ \\
\hline & Monoparental & 64 & $6,1 \%$ \\
\hline & Extensa & 85 & $8,1 \%$ \\
\hline & Ensamblada & 90 & $8,5 \%$ \\
\hline \multirow{4}{*}{ Trabajo padre } & Tiempo completo & 760 & $73,9 \%$ \\
\hline & Tiempo parcial & 170 & $16,5 \%$ \\
\hline & Desempleado & 50 & $4,9 \%$ \\
\hline & Jubilado o pensionista & 49 & $4,8 \%$ \\
\hline
\end{tabular}




\begin{tabular}{|c|c|c|c|}
\hline \multirow{4}{*}{ Trabajo madre } & Tiempo completo & 474 & $45,2 \%$ \\
\hline & Tiempo parcial & 309 & $29,5 \%$ \\
\hline & Desempleado & 238 & $22,7 \%$ \\
\hline & Jubilado o pensionista & 27 & $2,6 \%$ \\
\hline \multirow{7}{*}{ Estudios padre } & Sin estudios & 59 & $5,7 \%$ \\
\hline & Educación Primaria & 171 & $16,6 \%$ \\
\hline & E.S.O & 320 & $31,0 \%$ \\
\hline & Bachillerato/CFGM & 241 & $23,4 \%$ \\
\hline & CFGS/ Diplomatura & 109 & $10,6 \%$ \\
\hline & Licenciatura o grado & 106 & $10,3 \%$ \\
\hline & Doctorado & 26 & $2,5 \%$ \\
\hline \multirow{7}{*}{ Estudios madre } & Sin estudios & 43 & $4,1 \%$ \\
\hline & Educación Primaria & 149 & $14,2 \%$ \\
\hline & E.S.O & 301 & $28,7 \%$ \\
\hline & Bachillerato/ CFGM & 253 & $24,2 \%$ \\
\hline & CFGS/ Diplomatura & 142 & $13,6 \%$ \\
\hline & Licenciatura o grado & 134 & $12,8 \%$ \\
\hline & Doctorado & 25 & $2,4 \%$ \\
\hline
\end{tabular}

\section{Instrumento}

Los datos fueron recopilados a través del cuestionario ad hoc, "Evaluación de las prácticas de ocio familiar. Cuestionario para adolescentes" cuya a validez de contenido se establece a través de una revisión de la literatura existente y un panel de expertos de nueve cuestiones cualitativas en el que participaron 17 investigadores de ocio a nivel nacional e internacional. También se llevó a cabo una validación de interjueces, por parte de 10 docentes universitarios y cuatro trabajadores del ocio, reduciendo el número de ítems y modificando la redacción. Como resultado se obtuvo un cuestionario de 50 ítems distribuidos en cinco dimensiones. Esta investigación se centra en la dimensión de capacidades, en la que el participante indicaba si el ocio familiar contribuye o no al desarrollo de 12 capacidades específicas propuestas por los expertos y consensuadas por los jueces.

En lo que respecta a la consistencia interna se logró un coeficiente a de Cronbach de .959 en la totalidad del cuestionario y .985 en la dimensión de capacidades, considerándose bastante alta (González y Pazmiño, 2015). Además, y dado que el valor de la prueba de esfericidad de Bartlett fue significativo $(\mathrm{p}<, 000)$ y el coeficiente $\mathrm{KMO}$ alto $(, 757)$, se llevó a cabo la validez de constructo mediante un análisis factorial exploratorio, a partir del cual se obtuvieron 3 factores que explican el 60,336 de la varianza total, y cuyas cargas factoriales son superiores a .5 (Tabla 2).

De cara a las variables independientes también se tomó en consideración los datos identificativos referidos a las variables personales (edad, nacionalidad y titularidad de centro) y familiares (tipo de familia, situación laboral padres y nivel de estudios padres). 


\section{Tabla 2}

Matriz de factores rotados. Estructura factorial final, pesos factoriales, varianza explicada y fiabilidad

\begin{tabular}{|c|c|c|c|c|}
\hline Ítems & Comunalidad & F1 CO* & F2 CA* & $\mathrm{F}_{3} \mathrm{CR}^{*}$ \\
\hline C12. Capacidad crítica &, 595 & ,736 & & \\
\hline C11. Trabajo en equipo & ,493 & 696 & & \\
\hline C2.Capacidad de análisis & ,484 & ,684 & & \\
\hline C1 Planificación &, 551 & ,644 & & \\
\hline C8Convivencia &, 506 &, 590 & & \\
\hline C4.Creatividad & 674 & & 819 & \\
\hline C3.Atención & 678 & & ,769 & \\
\hline C6.Memoria & 669 & &, 768 & \\
\hline C10. Inteligencia emocional &, 560 & &, 519 & \\
\hline C7.Comunicación &, 743 & & & ,861 \\
\hline C9.Regulación del comportamiento & ,685 & & & 817 \\
\hline C5.Resolución de problemas & ,602 & & & ,620 \\
\hline \% de varianza & 60,33 & 36,30 & 13,57 & 10,46 \\
\hline a de Cronbach & ,985 & ,745 & ,773 &, 715 \\
\hline
\end{tabular}

*CO= Capacidades organizativas; $\mathrm{CA}=$ Capacidades académicas; $\mathrm{CR}=$ Capacidades relacionales

\section{Análisis de datos}

Se utilizó el programa estadístico SPSS versión 24 para realizar el análisis descriptivo (frecuencias y porcentajes) como el inferencial no paramétrico, según los resultados de la prueba de normalidad Kolmogorov-Smirnov ( $<<, 001)$, aplicando la prueba Chi-Cuadrado con un nivel de significación estadística de $\alpha=0,05$. Para determinar la magnitud de la asociación entre las categorías de las variables consideradas, se calculó el tamaño del efecto mediante el coeficiente de contingencia (CC) 


\section{Resultados}

A continuación, se muestran los resultados de la investigación atendiendo a los objetivos formulados en la misma

\section{Desarrollo de capacidades atribuibles al ocio familiar}

El desarrollo de las capacidades que se promueven en el ocio familiar ha sido expuesto en la Tabla 3 en función de las siguientes categorías: organizativas, académicas y relacionales, siendo ésta última la más fomentada, como evidencian los elevados porcentajes obtenidos en comunicación $(86,8 \%)$, regulación del comportamiento $(74,8 \%)$ y resolución de problemas $(72,9 \%)$. Entre las organizativas destaca la convivencia (89,1\%), siendo la más fomentada de todas las capacidades, seguida de la capacidad critica (72,8\%), mientras que, en las académicas, dimensión menos fomentada por el ocio familiar según la percepción de los adolescentes, destaca la inteligencia emocional $(74,1 \%)$ por encima del resto. Finalmente, la memoria (34,2\%), el trabajo en equipo (42,3\%), la creatividad (43,6\%) y la atención ( $46,9 \%)$, son las capacidades que se atribuyen en menor medida al ocio familiar.

\section{Tabla 3}

Frecuencias y porcentajes del desarrollo de capacidades a través del ocio familiar

\begin{tabular}{|c|c|c|c|c|c|}
\hline \multirow{2}{*}{ Factor } & \multirow{2}{*}{ Ítem } & \multicolumn{2}{|c|}{ Frecuencia } & \multicolumn{2}{|c|}{ Porcentaje } \\
\hline & & Sí & No & Sí & No \\
\hline \multirow{5}{*}{$\begin{array}{l}\text { Organizativ } \\
\text { as }\end{array}$} & C12.Capacidad crítica & 768 & 286 & $72,8 \%$ & $27,2 \%$ \\
\hline & C11.Trabajo en equipo & 446 & 608 & $42,3 \%$ & $57,7 \%$ \\
\hline & C2.Capacidad de análisis & 616 & 438 & $58,4 \%$ & $41,6 \%$ \\
\hline & C1.Planificación & 639 & 415 & $60,6 \%$ & $39,4 \%$ \\
\hline & C8.Convivencia & 939 & 115 & $89,1 \%$ & $10,9 \%$ \\
\hline \multirow{4}{*}{ Académicas } & C4.Creatividad & 460 & 594 & $43,6 \%$ & $56,4 \%$ \\
\hline & C3.Atención & 494 & 560 & $46,9 \%$ & $53,1 \%$ \\
\hline & C6.Memoria & 360 & 694 & $34,2 \%$ & $65,8 \%$ \\
\hline & C10.Inteligencia emocional & 728 & 273 & $74,1 \%$ & $25,9 \%$ \\
\hline \multirow{3}{*}{$\begin{array}{l}\text { Relacionale } \\
\mathrm{s}\end{array}$} & C7.Comunicación & 915 & 139 & $86,8 \%$ & $13,2 \%$ \\
\hline & $\begin{array}{l}\text { C9.Regulación } \\
\text { comportamiento }\end{array}$ & 788 & 266 & $74,8 \%$ & $25,2 \%$ \\
\hline & C5.Resolución de problemas & 768 & 286 & $72,9 \%$ & $27,1 \%$ \\
\hline
\end{tabular}

\section{Desarrollo de capacidades en función de las variables personales y familiares}

En la tabla 4 se muestran los coeficientes de significación arrojados por la prueba Chi Cuadrado de las variables personales de la edad, titularidad de centro y nacionalidad, y las variables familiares, tipo de familia, trabajo de los padres y estudios de los padres. En relación a la edad, la inteligencia emocional (C10), el trabajo en equipo (C11) y la planificación (C1) son las únicas capacidades que presentan diferencias significativas. Por el contrario, la titularidad 
del centro se relaciona significativamente con todas las capacidades analizadas, a excepción de: el desarrollo de la resolución de problemas ( $\left.\mathrm{C}_{5}\right)$, la comunicación ( $\mathrm{C} 7$ ), la convivencia ( $\mathrm{C} 8$ ) y la regulación del comportamiento (C9), según la percepción del adolescente. Por último, la nacionalidad resulta significativa para la percepción del desarrollo de la convivencia (C8) y la expresión de las emociones (C10).

En lo que respecta a las variables personales, contrariamente a lo esperado, no presentan ninguna significación como en el caso de los estudios del padre, o bien muestran una significación aislada con alguna capacidad. De modo que la atribución de la capacidad de atención (C3) al ocio familiar se relaciona significativamente con el tipo de familia; el trabajo en equipo (C11) con los estudios y la situación laboral de la madre; y finalmente, la resolución de problemas ( $\mathrm{C} 5$ ) con la situación laboral del padre.

\section{Tabla 4}

Estadísticos de significación desarrollo de las capacidades en relación a las variables personales y familiares

\begin{tabular}{|c|c|c|c|c|c|c|c|c|}
\hline Capacidades & Edad & $\begin{array}{l}\text { Titularidad } \\
\text { centro }\end{array}$ & Nacional. & $\begin{array}{l}\text { Tipo } \\
\text { de } \\
\text { familia }\end{array}$ & $\begin{array}{l}\text { Trabajo } \\
\text { padre }\end{array}$ & $\begin{array}{l}\text { Trabajo } \\
\text { madre }\end{array}$ & $\begin{array}{l}\text { Estudios } \\
\text { padre }\end{array}$ & $\begin{array}{l}\text { Estudios } \\
\text { madre }\end{array}$ \\
\hline $\begin{array}{l}\text { 12.Capacidad } \\
\text { crítica }\end{array}$ &, 317 &, $012^{*}$ & ,439 & ,779 &, 524 & ,074 &, 331 & ,730 \\
\hline $\begin{array}{l}\text { 11.Trabajo en } \\
\text { equipo }\end{array}$ &, $042 *$ &, $000 * *$ &, 525 & ,077 &, 527 &, $045^{*}$ & 101 & ,034* \\
\hline $\begin{array}{l}\text { 2.Capacidad de } \\
\text { análisis }\end{array}$ & ,920 &, $000 * *$ & ,184 & ,139 &, 541 & ,313 & 117 & ,378 \\
\hline 1.Planificación & ,010* & ,029* & ,943 & 179 & 351 & ,397 & ,626 & ,990 \\
\hline 8.Convivencia &, 307 & , 145 & ,027 & ,952 & ,884 &, 541 & ,179 & ,876 \\
\hline 4.Creatividad & ,989 &, $000 * *$ & ,278 & ,409 & ,323 & 189 &, 580 & ,963 \\
\hline 3.Atención & ,768 &, $000 * *$ & ,982 &, $044^{*}$ & ,639 & ,662 & ,894 & ,301 \\
\hline 6.Memoria &, 585 &, $000 * *$ & ,923 & ,243 & ,095 & ,078 & ,905 & ,803 \\
\hline $\begin{array}{l}\text { 10.Intelige. } \\
\text { emocional }\end{array}$ &, $030 *$ &, $011^{*}$ &, $008 * *$ &, 589 & ,895 & ,094 & ,454 & , 986 \\
\hline 7.Comunicación & 686 &, 720 & ,096 & ,132 & ,830 & ,444 & ,770 & ,593 \\
\hline $\begin{array}{l}\text { 9. Regulación } \\
\text { conducta }\end{array}$ & ,595 &, $016^{*}$ & ,855 & ,347 & ,119 & ,517 & ,874 & ,865 \\
\hline $\begin{array}{l}5 . \text { Resoluc. de } \\
\text { problemas }\end{array}$ & 619 & 168 & ,239 & 088 & ,029* &, 305 & 136 & 891 \\
\hline
\end{tabular}

$* * \mathrm{p}<, 000 * \mathrm{p}<, 005$

\section{Desarrollo de capacidades y la titularidad del centro}

Atendiendo a la significatividad, la titularidad de centro es la variable que guarda mayor relación con el listado de capacidades (tabla 5), por lo que se han realizado análisis pormenorizados de frecuencias y porcentajes. Desde una visión global, en todas las 
capacidades el alumnado de educación secundaria de los centros de titularidad privadaconcertada tienen una mayor identificación de las capacidades cuestionadas dentro de su ocio familiar.

\section{Tabla 5}

Frecuencias y porcentajes del desarrollo de capacidades en el ocio familiar, en función de la titularidad del centro

\begin{tabular}{|c|c|c|c|c|c|c|c|c|}
\hline & \multicolumn{4}{|c|}{ Frecuencia } & \multicolumn{4}{|c|}{ Porcentaje } \\
\hline & \multicolumn{2}{|c|}{ Público } & \multicolumn{2}{|c|}{$\begin{array}{l}\text { Privado- } \\
\text { Concertado }\end{array}$} & \multicolumn{2}{|c|}{ Público } & \multicolumn{2}{|c|}{$\begin{array}{l}\text { Privado- } \\
\text { Concertado }\end{array}$} \\
\hline & Sí & No & Sí & No & Sí & No & Sí & No \\
\hline 12. Capacidad crítica & 578 & 236 & 190 & 50 & $71,0 \%$ & $29,0 \%$ & $79,2 \%$ & $20,8 \%$ \\
\hline 11.Trabajo en equipo & 317 & 497 & 129 & 111 & $38,9 \%$ & $61,1 \%$ & $53,8 \%$ & $46,3 \%$ \\
\hline $\begin{array}{l}\text { 2.Capacidad } \\
\text { análisis }\end{array}$ & 448 & 366 & 168 & 72 & $55,0 \%$ & $45,0 \%$ & $70,0 \%$ & $30,0 \%$ \\
\hline 1.Planificación & 479 & 335 & 160 & 80 & $58,8 \%$ & $41,2 \%$ & $66,7 \%$ & $33,3 \%$ \\
\hline 8.Convivencia & 719 & 95 & 189 & 51 & $88,3 \%$ & $11,7 \%$ & $91,7 \%$ & $8,3 \%$ \\
\hline 4.Creatividad & 324 & 490 & 136 & 104 & $39,8 \%$ & $60,2 \%$ & $56,7 \%$ & $43,3 \%$ \\
\hline 3.Atención & 357 & 457 & 137 & 103 & $43,9 \%$ & $56,1 \%$ & $57,1 \%$ & $42,9 \%$ \\
\hline 6.Memoria & 249 & 565 & 111 & 129 & $30,6 \%$ & $69,4 \%$ & $46,3 \%$ & $53,8 \%$ \\
\hline 10.Intelig. emocional & 588 & 226 & 193 & 47 & $72,2 \%$ & $27,8 \%$ & $80,4 \%$ & $19,6 \%$ \\
\hline 7.Comunicación & 705 & 109 & 210 & 30 & $86,6 \%$ & $13,4 \%$ & $87,5 \%$ & $12,5 \%$ \\
\hline $\begin{array}{l}\text { 9. Regulación } \\
\text { conducta }\end{array}$ & 599 & 215 & 189 & 51 & $73,6 \%$ & $26,4 \%$ & $78,8 \%$ & $21,3 \%$ \\
\hline $\begin{array}{l}\text { 5. Resoluc. } \\
\text { problemas }\end{array}$ & 584 & 230 & 183 & 57 & $71,7 \%$ & $28,3 \%$ & $76,3 \%$ & $23,8 \%$ \\
\hline
\end{tabular}

Tal y como se muestra en la tabla 6, a pesar de que se halla diferencias significativas en tres cuartas partes de las capacidades incluidas en el estudio, ninguno coeficientes de contingencia obtiene valor típico 0,3 (Cohen, 1988). Por lo cual, se afirma que no hay una alta magnitud en las diferencias identificadas.

\section{Tabla 6}

Significación estadística y coeficientes de contingencia del desarrollo de capacidades en el ocio familiar en función de la titularidad del centro

\begin{tabular}{lllll}
\hline \multicolumn{4}{c}{ Público/ Concertado-Privado } \\
\hline & $X^{2}$ & Sig & $\begin{array}{l}\text { Coeficiente } \\
\text { contingencia }\end{array}$ & de \\
\hline 12.Capacidad crítica & 6,241 &, $012^{*}$ &, 077
\end{tabular}




\begin{tabular}{llll} 
11.Trabajo en equipo & 16,647 &, $000^{* *}$ &, 125 \\
2.Capacidad de análisis & 17,087 &, $000^{* *}$ &, 126 \\
1.Planificación & 4,750 &, $029^{*}$ &, 067 \\
8.Convivencia & 2,124 &, 145 & - \\
\hline 4.Creatividad & 21,430 &, $000^{* *}$ &, 141 \\
3.Atención & 13,02 &, $000^{* *}$ &, 110 \\
6.Memoria & 21,430 &, $000^{* *}$ &, 141 \\
10.Inteligencia emocional & 6,463 &, $011^{*}$ &, 078 \\
\hline 7.Comunicación &, 128 &, 720 & - \\
9.Regulación conducta & 2,618 &, $016^{*}$ &, 050 \\
5.Resoluc. de problemas & 1,899 &, 168 & - \\
\hline
\end{tabular}

** $p<, 000 * p<, 05$

\section{Discusión y conclusiones}

Aunque el ocio familiar es una experiencia mutuamente positiva y beneficiosa para todos los miembros de la familia, generalmente se aborda desde lo abstracto-universal, negando la multiplicidad de significados, experiencias, factores, beneficios y limitaciones que pudieran ocurrir (Trussell et al., 2017). Este estudio muestra, de forma global, que los adolescentes consideran el ocio familiar como un recurso adecuado para el desarrollo de diversas y variadas capacidades. De hecho, el ocio familiar se concibe como una escuela de la vida donde se adquieren habilidades relacionales, sociales y emocionales vitales para la vida en comunidad (Kang et al., 2019; Townsend et al., 2017) y representa una excelente oportunidad para adquirir diferentes capacidades mediante el ejercicio de una práctica positiva y de calidad (Melton et al., 2020).

Atendiendo a los factores arrojados estadísticamente, las capacidades relacionales y organizativas prevalecen sobre las académicas, lo que indica que el ocio familiar con fines educativos se encuentra escasamente potenciado frente a otras modalidades, tal y como manifiestan estudios previos (Martín et al., 2018). De hecho, la significatividad de la titularidad del centro abarca la totalidad de las capacidades académicas, algo no atribuible a las variables familiares contempladas, cuando curiosamente el juego ha sido tradicionalmente enmarcado en el ámbito informal. De este modo, se corre el peligro de establecer una brecha entre el acompañamiento académico y la diversión, olvidando que existen multitud de juegos familiares que potencian la atención, memoria, creatividad e inteligencia emocional (Véase a clasificación de juegos aportada por Koehler et al. 2016). Aunque existen evidencias de una fuerte relación entre el ocio familiar y las habilidades académicas de los adolescentes (Badura et al, 2016; Hernández-Prados y Álvarez-Muñoz, 2019), hay que fomentar la consciencia parental al respecto.

De todas las capacidades que fomenta el ocio familiar destaca la convivencia y la comunicación. Impulsar dinámicas lúdicas colectivas favorece la convivencia y el hogar se convierte en entorno caracterizado por la armonía y amabilidad (Costa et al., 2020), fortaleciendo, a su vez, las relaciones humanas que se vuelven más cercanas, afectivas y satisfactorias (Sanz et al., 2018). Del mismo modo, potencia otras capacidades organizativas como el trabajo en equipo, sentimiento de pertenencia, planificación y análisis crítico, 
especialmente cuando el ocio familiar constituye un producto común y no una imposición (Shannon, 2017).

Por otro lado, la capacidad de trabajar colaborativamente se relaciona significativamente con la edad, la titularidad del centro, los estudios y trabajo de la madre, por lo que podemos afirmar el papel protagónico de ésta en la transmisión del valor de la cooperación. Desde una perspectiva histórica, la mujer ha sido invisibilizada, limitando su participación en la mayoría de experiencias de ocio (Merelas y Caballo, 2018), exceptuando el ocio familiar donde su presencia ha sido notoria (Townsend et al., 2017) y la masculina, generalmente, puntual y esporádica.

El ocio familiar también supone una excelente oportunidad para consolidar la comunicación entre los miembros familiares (O'Neill et al., 2017), fundamentalmente cuando se desarrolla en el hogar fruto de la espontaneidad (Townsend et al., 2017). De hecho, estas habilidades comunicativas configuran un entorno familiar seguro y saludable. El ocio familiar también contribuye a desarrollar la capacidad de regulación de la conducta, aspecto esencial para mantener relaciones saludables (Melton, 2017), además de aprender el sistema de normas y valores que modulan la conducta y permite la resolución pacífica de los conflictos, convirtiendo las diferencias y discrepancias en oportunidades para el crecimiento personal y familiar (Costa et al., 2020).

Lejos de lo esperado, pues existen diferencias significativas en función de la edad, especialmente entre adultos y menores (Hodge et al., 2017), aunque el mapa de conocimiento al respecto es escaso para establecer generalizaciones consistentes (Aaltonen et al., 2015) y evidencias del peso del género en los estudios de ocio familiar (Trussell et al, 2017), en lo que respecta al desarrollo de las capacidades la percepción de los adolescentes no se ve condicionada por estas variables. Aún así, existen estudios que sostienen que la gestión y control emocional aumenta con la edad (Chen et al., 2020), empeora con el incremento de la autoexigencia y los complejos (keefer et al., 2013) y tiene un fuerte componente cultural, asociando esta capacidad del ocio familiar a la nacionalidad (Hodge et al., 2017; Pongrac et al., 2019)

Los resultados obtenidos evidencian que la titularidad del centro es la variable estrella en las capacidades del ocio familiar, a pesar del bajo tamaño del efecto. Coincidiendo con otros estudios en los que la comunicación, el sentimiento de pertenencia y modelos de ocio se relacionan significativamente con dicha variable (Hodge et al., 2017). Los adolescentes de los centros educativos concertados-privados perciben su ocio familiar como un producto más fructífero de cara al desarrollo de capacidades de diferente naturaleza. Probablemente porque el mayor poder adquisitivo de las familias de los centros concertados-privados posibilita el acceso a un ocio familiar más diversificado y enriquecedor (Khoo y Yihjun, 2020). Para Coleman (2019) esta diferencia radica en el componente religioso de algunos de los centros de esta titularidad, ya que promueven numerosas iniciativas en las que participan la totalidad de las familias de su alumnado.

Mas allá de los logros estadísticos que muestra esta investigación, se debe resaltar a los adolescentes como población participante, ya que como afirman Pomfret y Varley (2019) los estudios de ocio familiar, especialmente los vinculados al turismo, suelen investigar el punto de vista de los progenitores. Aunque no se descarta la posibilidad de un estudio comparativo de la atribución de capacidades al ocio que realizan diferentes miembros de la unidad familiar. Así mismo se aporta un instrumento fiable y valido que no se limita al contenido de la actividad o connotaciones de frecuencia y tiempo (Martín et al., 2018; Melton y Ellis, 2019) y proporciona una clasificación de 12 capacidades atribuibles al ocio familiar según la percepción de los adolescentes, agrupadas en tres categorías: organizativas, académicas y 
relacionales. También presenta limitaciones, principalmente metodológicas, ya que el carácter transversal hace imposible dictaminar la magnitud de los hallazgos posteriores, algunos adolescentes no cumplimentaron adecuadamente el cuestionario, reduciendo la magnitud de la muestra, y quedaron sin explorar otras variables como la etapa educativa.

A modo de conclusión, lo relacional y organizativo está más relacionado con las prácticas de ocio que lo academicista de manera que se consolida el papel del ocio familiar como contexto educativo informal donde se desarrollan aprendizajes, pero alejados de lo que es prioritario en los contextos formales. Sin embargo, el distanciamiento entre ambos contextos es contraproducente. Debería entenderse la vinculación escuela y familia como entornos vinculados-no diferenciados, donde lo que concurre en un espacio es factor de condicionamiento para el otro (Gomáriz et al., 2017). Sin perder de vista el generar un tiempo de disfrute colectivo en el que participe toda la familia, se debe fomentar desde los diferentes agentes promotores del ocio familiar experiencias alternativas globalizadoras que enriquezcan también el desarrollo de capacidades académicas.

\section{Referencias}

Aaltonen S., Latvala A., Rose R. J., Kujala U. M., Kaprio J., y SilventoinenK. (2016). Leisure-time physical activity and academic performance:Cross-lagged associations from adolescence to young adulthood. Scientificreports, 6, 39215. https://doi.org/10.1038/srep39215

Alves, A. F., Gomes, C. M. A., Martins, A., y da Silva Almeida, L. (2017). Cognitive performance and academic achievement: How do family and school converge?. European Journal of Education and Psychology, 10(2), 49-56. https://doi.org/10.1016/j.ejeps.2017.07.001

Badura, P., Sigmund, E., Madarasova Geckova, A., Sigmundova, D., Sirucek, J., van Dijk, J. P., y Reijneveld, S. A. (2016). Is participation in organized leisure-time activities associated with school performance in adolescence?. PLoS One, 11(4), e0153276. https://doi.org/10.1371/journal.pone.0153276

Boukamel, O., Emery, Y., y Gieske, H. (2019). Towards an Integrative Framework of Innovation Capacity. The Innovation Journal, 24(3). https://serval.unil.ch/resource/serval:BIB_61EF194278EB.P001/REF.pdf

Chen, C., Yuan, Z., y Zhu, H. (2020). Playing, parenting and family leisure in parks: Exploring emotional geographies of families in Guangzhou Children's Park, China. Children's Geographies, 18(4), 463-476. https://doi.org/10.1080/14733285.2019.1676879

Cohen, J. (1988). Statistical power analysis for the behavioral science (2nd ed.). Lawrence Erlbaum Associates.

Coleman, J. S. (2019). Family involvement in education. Routledge.

Costa, F. M., Pereira, A. P., y Araujo, N. (2020). The experience of family leisure. Revista da SPAGESP, 21(2), 97-110. http://pepsic.bvsalud.org/pdf/rspagesp/v21n2/v21n2a08.pdf

Fernández-Mesa, A., Alegre-Vidal, J., y Chiva-Gómez, R. (2012). Orientación emprendedora, capacidad de aprendizaje organizativo y desempeño innovador. Journal of technology management \& innovation, 7(2), 157-170. http://dx.doi.org/10.4067/S071827242012000200013

Gallego, L., y Araque, O. (2019). Variables de influencia en la capacidad de aprendizaje. un análisis por conglomerados y componentes principales. Información tecnológica, 30(2), 257-264. http://dx.doi.org/10.4067/S0718-07642019000200257 
Gomáriz Vicente, M. A., Hernández Prados, M. Á., García Sanz, M. P., y Parra Martínez, J. (2017). Tejiendo puentes entre la escuela y la familia. El papel del profesorado. Bordón. Revista de pedagogía, 69(2), 41-57. https://doi.org/10.13042/Bordon.2016.49832

González Alonso, J., y Pazmiño Santacruz, M. (2015). Cálculo e interpretación del Alfa de Cronbach para el caso de validación de la consistencia interna de un cuestionario, con dos posibles escalas tipo Likert. Revista publicando, 2(1), 62-67. https://nbnresolving.org/urn:nbn:de:0168-ssoar-423821

Hernández Prados, M. Á., y Álvarez Muñoz, J. S. (2019). Family leisure and academic achievement. Perception of the families. Italian journal of educational research, (23), 86-105. https://ojs.pensamultimedia.it/index.php/sird/article/view/3685

Hernández, R., Fernández, C., y Baptista, P. (2010). Metodología de la Investigación. McgrawHILL

Hodge, C. J., Duerden, M. D., Layland, E. K., Lacanienta, A., Goates, M. C., y Niu, X. M. (2017). The association between family leisure and family quality of life: $A$ meta-analysis of data from parents and adolescents. Journal of Family Theory \& Review, 9(3), 328-346. https://doi.org/10.1111/jftr.12202

Hodge, C. J., Kanters, M. A., Forneris, T., Bocarro, J. N., y Sayre-McCord, R. (2017). A Family Thing: Positive Youth Development Outcomes of a Sport-Based Life Skills Program. Journal of Park \& Recreation Administration, 35(1). https://doi.org/10.18666/JPRA-2017V35-11-6840

Kang, S., Lee, W. S., Moon, J., y Kim, W. G. (2019). The influence of leisure and travel experiences on the school enjoyment of adolescents: a panel analysis. Leisure Studies, 38(4), 548-557. https://doi.org/10.1080/02614367.2019.1586980

Keefer, K. V., Holden, R. R., y Parker, J. D. (2013). Longitudinal assessment of trait emotional intelligence: measurement invariance and construct continuity from late childhood to adolescence. Psychological assessment, 25(4), 1255. https://psycnet.apa.org/doi/10.1037/a0033903

Khoo-Lattimore, C., y Jihyun Yang, M. (2020). The constructions of family holidays in young middle-class Malaysian Chinese children. Journal of China Tourism Research, 16(1), 6277. https://doi.org/10.1080/19388160.2018.1513884

Koehler, M. J., Greenhalgh, S. P., y Boltz, L. O. (2016). Here we are, now entertain us! A comparison of educational and non-educational board games. In Society for Information Technology \& Teacher Education International Conference (pp. 567-572). Association for the Advancement of Computing in Education (AACE).

Martín Quintana, J. C., Alemán Falcón, J., Calcines Piñero, M. A., y Izquierdo Clemente, D. (2018). Analysis of family shared leisure time in early childhood and their relation with parental competencies. Early Child Development and Care, 188(11), 1580-1592. https://doi.org/10.1080/03004430.2018.1499097

McCormick, M. P., Weissman, A. K., Weiland, C., Hsueh, J., Sachs, J., y Snow, C. (2020). Time well spent: Home learning activities and gains in children's academic skills in the prekindergarten year. Developmental psychology, 56(4), 710. https://psycnet.apa.org/doi/10.1037/dev0000891 
Merelas, T., y Caballo, M. B. (2018). Enfoques feministas sobre los tiempos de ocio de las mujeres. En A. Madariaga, y A. Ponce de León, Ocio y participación social en entornos comunitarios (101-117). Universidad de La Rioja.

Melton, K. K. (2017). Family activity model: Crossroads of activity environment and family interactions in family leisure. Leisure Sciences, 39(5), 457-473. https://doi.org/10.1080/01490400.2017.1333056

Melton, K. K., y Ellis, G. D. (2019). Measurement of time involvement in family activities: Alternative scaling procedures for the family leisure activity profile. Leisure Sciences, 41(6), 535-541. https://doi.org/10.1080/01490400.2018.1458669

Melton, K. K., Hodge, C. J., y Duerden, M. D. (2020). Ecology of family experiences: Contextualizing family leisure for human development \& family relations. Journal of Leisure Research, 1-20. https://doi.org/10.1080/00222216.2020.1802374

O'Neill, T., Mandak, K., y Wilkinson, K. M. (2017, September). Family leisure as a context to support augmentative and alternative communication intervention for young children with complex communication needs. Seminars in speech and language, 38 (4), 313- 320. https://doi.org/10.1055/s-0037-1604278

Park, H., y Lee, K. S. (2020). The association of family structure with health behavior, mental health, and perceived academic achievement among adolescents: a 2018 Korean nationally representative survey. BMC public health,20(1), 1-10. https://doi.org/10.1186/s12889-020-08655-z

Pomfret, G., y Varley, P. (2019). Families at leisure outdoors: well-being through adventure. Leisure studies, 38(4), 494-508. https://doi.org/10.1080/02614367.2019.1600574

Pongrac, J., Mohorić, T., y Anić, P. (2019). Gender and national differences in emotional intelligence and empathy: Comparison of Croatian and Portuguese samples. Horizons of Psychology, 28, 19-27. https://doi.org/10.20419/2019.28.497

Sanz Arazuri, E., Saenz De Jubera Ocon, M., y Cano, R. (2018). Attitudes of parents and children towards shared family leisure. Pedagogía Social, (32), 53-64. https://doi.org/10.7179/PSRI2018.32.05

Shannon, C. S. (2017). Family leisure and leisure service provision: Making the case for including perspectives from practice. Leisure Sciences, 39(5), 426-435. https://doi.org/10.1080/01490400.2017.1333054

Townsend, J. A., Van Puymbroeck, M., y Zabriskie, R. B. (2017). The core and balance model of family leisure functioning: A systematic review. Leisure Sciences, 39(5), 436-456. https://doi.org/10.1080/01490400.2017.1333057

Trussell, D. E., Jeanes, R., y Such, E. (2017). Revisiting family leisure research and critical reflections on the future of family-centered scholarship. Leisure Sciences, 39(5), 385399. https://doi.org/10.1080/01490400.2017.1333059

Varela, L. y Maroñas, A. (2019). El ocio familiar del alumnado de educación secundaria obligatoria en Galicia. Bordón. Revista de pedagogía, 71(4), 135-150. https://doi.org/10.13042/Bordon.2019.68380

Zhou, L., Shen, H., Wu, M. Y., Wall, G., y Shen, X. (2019). Benefits of visiting heritage museums: Chinese parents' perspectives. International Journal of Heritage Studies, 25(6), 565581. https://doi.org/10.1080/13527258.2018.1428667 